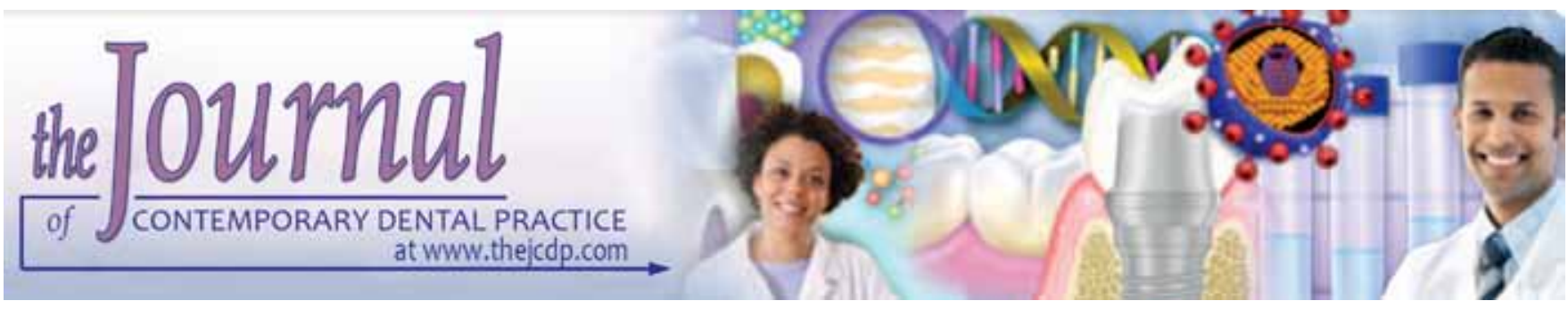

\title{
Effect of Energy Drinks on the Surface Texture of Nanofilled Composite Resin
}

\section{Khalid H Al-Samadani}

\section{ABSTRACT}

Objective: To study the effect of three energy drinks on the surface roughness of nanofilled composite resins after different periods of aging time.

Materials and methods: Composite resin disks, $6 \mathrm{~mm}$ diameter, $3 \mathrm{~mm}$ thickness were prepared from Filtec Z350 XT, Tetric EvoCeram and Filtec Z250 XT. Specimens fr/8om each material were tested after aging with Red Bull, Bison and Power Horse energy drinks and distilled water as a control. Specimens were stored at $37^{\circ} \mathrm{C}$ in dark containers for 1,3 and 6 months. Surface roughness Ra was assessed using a surface scanning interferometry before and after each storage period. Surface roughness differences $\Delta \mathrm{Ra}$ and $\mathrm{Ra}$ among specimens were measured. Mean values were statistically analyzed using multiple repeated measured (ANOVA), variance and multiple comparisons of the mean values were done with Bonferroni test, with $p<0.05$ as significance level.

Results: The surface roughness degradation change $\Delta \mathrm{Ra}$ and $\mathrm{Ra}$ value before and after occurred by Red Bull, Bison and Power Horse energy drinks was significantly different for all tested materials at all three times $p<0.05$. All specimens showed increase in surface roughness changes after aging periods of 1,3 and 6 months. The highest total surface roughness difference $\Delta \mathrm{Ra}$ and $\mathrm{Ra}$ was found in the Red Bull group after 6 months.

Conclusion: Energy drinks used in this study had surface degradation effect on the tested composite resin materials. The surface roughness increased with aging time however, it was clinically acceptable in all test groups after 6 months. The effect of energy drinks solutions on the surface roughness parameter of resin composites depends on type of solution and its acidity contents.

Keywords: Energy drinks, Aging, Surface roughness, Nanofilled composite resins.

How to cite this article: Al-Samadani $\mathrm{KH}$. Effect of Energy Drinks on the Surface Texture of Nanofilled Composite Resin. J Contemp Dent Pract 2013;14(5):830-835.

Source of support: Nil

Conflict of interest: None declared

\section{INTRODUCTION}

Composite resin is being used widely as an esthetic restorative material in anterior as well as posterior teeth. ${ }^{1,2}$ The addition of filler to the resin matrix of restorative materials increase the strength, the toughness under strain and the wear resistance of composites, all of which are fundamental to the durability of composite restoration. ${ }^{3}$ The restorative composite resins are generally classified according to the size, content and filler type such as bariumaluminum-silicate, lithium boro-barium glass, ytterbium trifluoride, quartz, zircon and silicon oxide. Studies have shown that the filler size and shape can influence the surface roughness of dental composites. ${ }^{3}$

Red Bull was introduced in Austria in 1987 and in the USA in 1997. Since then the energy beverages have grown exponentially. Hundreds of different brands are now marketed. The USA is the world's largest consumer of energy beverage by volume, roughly 290 million gallons beverages were consumed in $2007 .^{4}$

Resin composites material properties such as color, cavity adaptability and wear are important for clinical use. For example, in the oral cavity environment, dental cement is immersed in various aqueous solutions and clinical observations have shown that it under goes continuous erosion over time. One of the most important properties that determine the durability of dental materials in the oral cavity is their resistance to dissolution or disintegration. ${ }^{5}$

Studies have identified the consumption of acidic carbonated and noncarbonated beverages as one of the main cause of dental erosion. ${ }^{6}$ The roughening of the surface caused by wear and chemical degradation may also affect gloss and consequently decrease the occlusal height of the composite resin filled tooth. ${ }^{7}$ Consumption of energy beverages has been shown to produce dental erosion intraorally. Attempts to decrease the erosive 
potential of beverages have been made by adding calcium, increasing their $\mathrm{pH}$ or adding ingredients, such as casein phosphopeptide-stabilized amorphous calcium phosphate. ${ }^{8}$

The purpose of the study was to assess erosive potential of Red bull, Bison and Power Horse energy beverages.

\section{MATERIALS AND METHODS}

Three different solutions of energy drinks (sports beverages), Red Bull, Bison, Power Horse and Distilled water as control were evaluated and their effects on the surfers roughness of Filtek Z350 XT nanofilled (3M ESPE, dental products D-82229 Seefeld- Germany). Tetric EvoCeram Nanohybrid composite and Filtek Z250 XT Nanohybrid (3M ESPE, products D-82229 Seefeld- Germany) composite resin materials were, aged for 1,3 and 6 months were stored at $37^{\circ} \mathrm{C}$. The resin composite materials and energy drinks used in this study are shown in Table 1. Eighty specimens of resin composite materials were prepared in which five specimens for each material of different aging energy beverages (groups).

All specimens of the materials were dispended, manipulated, and polymerized according to the manufacturer's instructions. Teflon molds, measuring $6 \mathrm{~mm}$ internal diameter and $3 \mathrm{~mm}$ height were used to produce the specimens. A glass microscope slide, overlaid with a cover glass $22 \times 22 \mathrm{~mm}$ (BDH Borosilicate glass) to act as a separator, was placed at each open end of the mold. Their dual function was to provide compaction of the materials into a flat surface and to act as a separator between the mold and the glass microscope slide. The layering technique was employed, especially in the preparation of the specimens of the light-activated dental restorative materials. This technique entailed incremental filling of the mold and compacting with a plastic spatula, after which the composite material was irradiated with a 20 s pulse from a light-curing unit LED light emitting diode (3M ESPE, Dental products D-82229 Seefeld- Germany). The mold was completely filled with the material using this step-wise method, and was irradiated at each stage with 20s light pulses, with the light tip approximately $1 \mathrm{~mm}$ away from the specimens were kept dry at room temperature until all specimens were fabricated.

For the purpose of surface standardization, all specimens were polished to obtain a clinical finish while in the Teflon disk, with 3M Sof-Lex disks. The specimens while in the Teflon disk were labelled and stored in a $25 \mathrm{ml}$ HDPE wide mouth bottled filled with distilled water at $37^{\circ} \mathrm{C}$ for 24 hours (Bibby Sterilin Ltd, Stone, Staffs., England). The rehydration simulated the first day of service for provisional restorations in the oral environment. It is known that the type of materials used in this study imbibe most of the water during the first day of immersion. ${ }^{9-11}$

A standard amount of energy solution of aging medium (sport beverages) was prepared for all groups from $100 \mathrm{ml}$ of each type of energy drinks according to the suggestion of manufacturers. Among the solution of all groups, group A, was Red Bull, group B Bison, group C Power Horse and group D Distil water (control).

All the specimens were immersed in the energy drinks beverages of different groups to evaluate the surface roughness of the resin composite materials at different time interval. The 20 specimens of the each three resin composite materials were immersed in the solution of the groups A, B, C and D (control), for the periods of 1, 3 and 6 months at $37^{\circ} \mathrm{C}$.

The surface roughness profile of all specimens measured before immersing in different four groups were measured before exposure (baseline) with a surface scanning interferometry (Contour GT-K0 BRUKER - USA) Since surface roughness were being tested, before each measurements session, the surface scanning interferometry was calibrated according to the manufacturers' recommendations. And the surface Ra parameter was selected from many other parameters, where $\mathrm{Ra}$ is the arithmetical mean roughness of all the values of the Ra profile within the measuring length.

\begin{tabular}{llcc}
\multicolumn{2}{c}{ Table 1: Composite resin materials and energy drinks used in this study } \\
\hline Material & Type & Shade & Manufacturer \\
\hline Filtek Z350 XT & Universal Nanocomposite & A2 & 3M ESPE Dental Products D-82229 Seefeld -Germany. \\
Tetric EvoCeram & Universal Nanohybrid composite & A2 & Ivoclar Vivadent AG, FL-9494 Schaan \\
Filtek Z250 XT & $\begin{array}{l}\text { Nanohybrid composite } \\
\text { Red Bull }\end{array}$ & A2 & 3M ESPE D-82229 Seefeld - Germany \\
& $\begin{array}{l}\text { Energy drink (Sucrose, glucose, acidity } \\
\text { regulatory sodium, caffeine, vitamins, }\end{array}$ & - & Red Bull GmbH, Austria \\
nison & $\begin{array}{l}\text { natural flavors, colors) } \\
\text { Energy drink (carbonated water, sugar, }\end{array}$ & - & Abuljadayal Beverages INC, Jeddah, Saudi Arabia \\
& $\begin{array}{l}\text { citric acid, caffeine, sodium benzoate, } \\
\text { vitamins, natural flavors, colors) }\end{array}$ & & \\
P Horse & $\begin{array}{l}\text { Energy drink (Carbonated water, } \\
\text { sucrose, glucose, citric acid, taurine, }\end{array}$ & - & S.Spitz GmbH, Attnang-Puchheim, Austria \\
& caffeine, color, inositol, niacin, & & \\
pantothenic acid, vitamins) & & \\
\hline
\end{tabular}


Following the removal of the specimens from the aging solutions in all groups, they were dipped in a cleansing solution, consisting of $10 \mathrm{ml}$ soap and $900 \mathrm{ml}$ distilled water, moved up and down 10 times, and subsequently flushed with running tap water. Then the specimens were dipped in distilled water and rinsed by moving them up and down 10 times. Excess fluid on the surface was removed using a tissue paper. After surface roughness measurements at the time intervals indicated, the specimens were re-immersed in fresh solutions.

Measurements were repeated 5 times for each specimen, the surface roughness measurement was done at baseline (before) and at a time intervals of 1, 3 and 6 months.

And the mean values of the Ra (before and after at each period for all groups) and $\Delta \mathrm{Ra}$ data were calculated. The $\Delta$ Ra surface roughness difference was calculated from the mean of Ra before and after aging in deferent periods values for each specimen.

The mean estimated from the specimens of the materials aged with the energy beverages groups at different interval times were statistically analyzed. Mean values of the different groups at different aging times were compared using analysis of multiple repeated measured ANOVA variance and multiple comparisons of the mean values were done with Bonferroni test. In present study, $\mathrm{p}<0.05$ was considered as the level of significance.

\section{RESULTS}

Surface roughness of each nanofilled resin composites used in this study affected with aging time duration and different energy drinks (sport beverages) Red Bull, Bison, Power Horse and Distal water control was observed in the present study.

The mean values of the surface roughness changes $\Delta \mathrm{Ra}$ and $\mathrm{Ra}$ parameters before and after immersion in the energy drinks for all materials aged in a periods of 1 , 3 and 6 months, after exposure to the different types of Red Bull, Bison, Power Horse and Distal water as control are summarized in Table 2 and graphically represented in Figures 1 to 3 .

The total surface roughness differences $\Delta \mathrm{Ra}$ and surface roughness Ra after aging for all materials in a period of 1, 3 and 6 months with the three different types of energy drinks Red Bull, Bison, Power Horse and Distal water as a control were analyzed statistically using multiple repeated measured analysis of variance (ANOVA) followed by the Bonferroni multiple comparison tests at $p<0.05$ level of significance. It was found that there were highly significant differences in surface roughness Ra changes between materials at the three different aging periods of times for each aging solutions $(p<0.05)$ (Table 2$)$.

The surface roughness changes of the materials in a periods of 1,3 and 6 months exhibited by all aging energy drinks solutions Red Bull, Bison, Power Horse and the Dist. water (control) was highly significantly different. The total surface roughness $\Delta \mathrm{Ra}$ and $\mathrm{Ra}$ after aging shows that all materials had surface degradation with the increase of the surface roughness with the aging with the energy solutions and the surface roughness $\mathrm{Ra}$ increase with the increasing of the aging time (Figs 1 to 3 ).

\begin{tabular}{|c|c|c|c|c|c|c|c|c|c|c|}
\hline \multicolumn{11}{|c|}{ Surface roughness Ra in $\mathrm{nm}$ (Red Bull medium) } \\
\hline \multirow[b]{2}{*}{ Duration } & \multicolumn{3}{|c|}{$Z 350 \times T$} & \multicolumn{3}{|c|}{$Z 250 X T$} & \multicolumn{3}{|c|}{ Tetric EvoCream } & \\
\hline & Ra before & Ra after & $\begin{array}{c}\Delta R a \\
\text { diff }\end{array}$ & Ra before & Ra after & $\begin{array}{c}\Delta R a \\
\text { diff }\end{array}$ & Ra before & Ra after & $\begin{array}{c}\Delta R a \\
\text { diff }\end{array}$ & \\
\hline 1 month & 73.6 & 174.5 & 100.9 & 145.07 & 143.8 & -1.27 & 149.4 & 211.01 & 61.61 & \\
\hline 3 months & 73.6 & 199.3 & 125.7 & 145.07 & 148.5 & 3.43 & 149.4 & 230.9 & 81.5 & $p<0.00$ \\
\hline 6 months & 73.6 & 217.6 & 144 & 145.07 & 155.8 & 10.73 & 149.4 & 277.2 & 127.8 & \\
\hline \multicolumn{11}{|c|}{ Surface roughness $R a$ in $\mathrm{nm}$ (Bison medium) } \\
\hline 1 month & 73.6 & 89.6 & 16 & 145.07 & 146.1 & 1.03 & 149.4 & 153.2 & 3.8 & \\
\hline 3 months & 73.6 & 103.4 & 29.8 & 145.07 & 145.8 & 0.73 & 149.4 & 166.02 & 16.62 & $p<0.00$ \\
\hline 6 months & 73.6 & 117.0 & 43.45 & 145.07 & 148.9 & 3.83 & 149.4 & 167.9 & 18.5 & \\
\hline \multicolumn{11}{|c|}{ Surface roughness $\mathrm{Ra}$ in $\mathrm{nm}$ (Power Horse medium) } \\
\hline 1 month & 73.6 & 128 & 54.4 & 145.07 & 142.9 & -2.17 & 149.4 & 148.1 & -1.3 & \\
\hline 3 months & 73.6 & 132.04 & 58.44 & 145.07 & 144.7 & -0.37 & 149.4 & 144.06 & -5.34 & $p<0.00$ \\
\hline 6 months & 73.6 & 140.0 & 66.4 & 145.07 & 141.3 & -3.77 & 149.4 & 139.5 & -9.9 & \\
\hline \multicolumn{11}{|c|}{ Surface roughness Ra in $\mathrm{nm}$ (Dist. water medium) } \\
\hline 1 month & 73.6 & 77.4 & 3.8 & 145.07 & 145.08 & 0.01 & 149.4 & 150.01 & 0.61 & \\
\hline 3 months & 73.6 & 77.03 & 3.43 & 145.07 & 145.2 & 0.13 & 149.4 & 149.9 & 0.5 & $p<0.00$ \\
\hline 6 months & 73.6 & 78.02 & 4.42 & 145.07 & 144.07 & -1.0 & 149.4 & 150.5 & 1.1 & \\
\hline
\end{tabular}




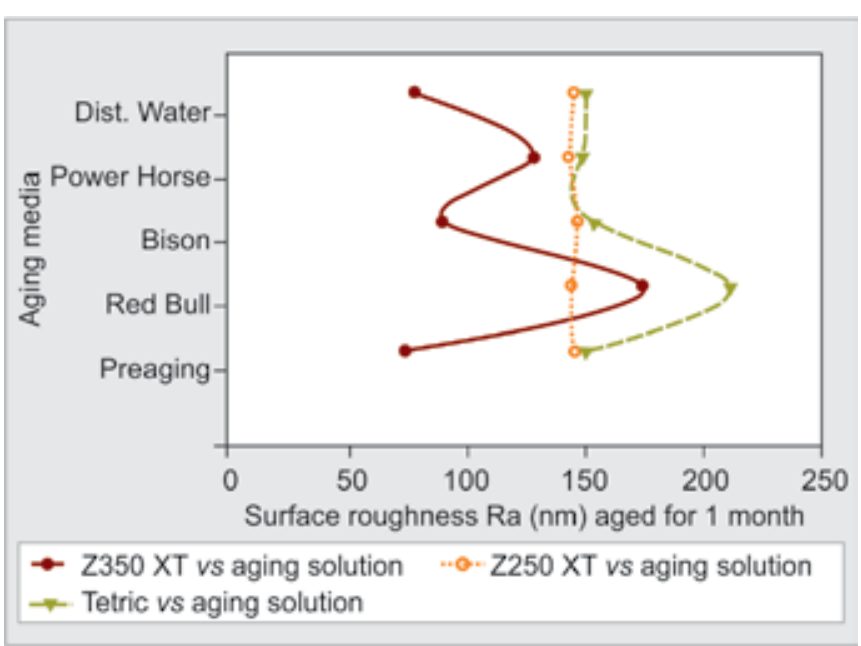

Fig. 1: Surface roughness $\mathrm{Ra}(\mathrm{nm})$ parameter pre- and postaged for 1 month with energy drinks for nanofilled composite resin

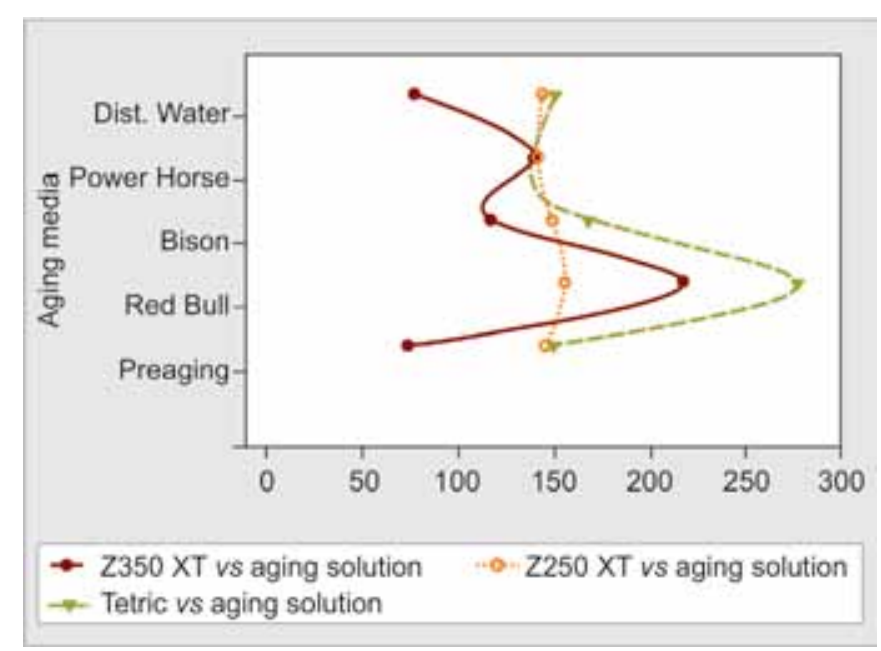

Fig. 3: Surface roughness $\mathrm{Ra}(\mathrm{nm})$ parameter pre- and postaged for 6 months with energy drinks for nanofilled composite resin

Among all the tested composite materials, it was consistently observed that Tetric EvoCeram specimens which were immersed in Power Horse and Z250 $\mathrm{XT}$ specimens immersed in Red Bull and Bison for 1 month revealed the lowest $\Delta \mathrm{Ra}$ values and the surface roughness is decreasing after 6 months, whereas the highest $\Delta \mathrm{Ra}$ values were observed in the Z350 XT specimens after being immersed in Red Bull for 6 months. When comparing the three composite materials immersed in Red Bull, shows highly significant differences were observed after immersion for periods of 1,3 and 6 months $(\mathrm{p}<0.000)$. Significant differences were observed for the materials Z350 XT, Tetric EvoCeram and Z250 XT after the period of 1,3 and 6 months in the specimens immerzed in Red Bull, Bison and Power Horse sports drinks, $\mathrm{p}<0.000$, respectively (Table 2 ). For specimens immerzed in distilled water, significant surface roughness Ra difference was observed between the materials after 6 months $(\mathrm{p}<0.000)$. All tested materials revealed an increase in the

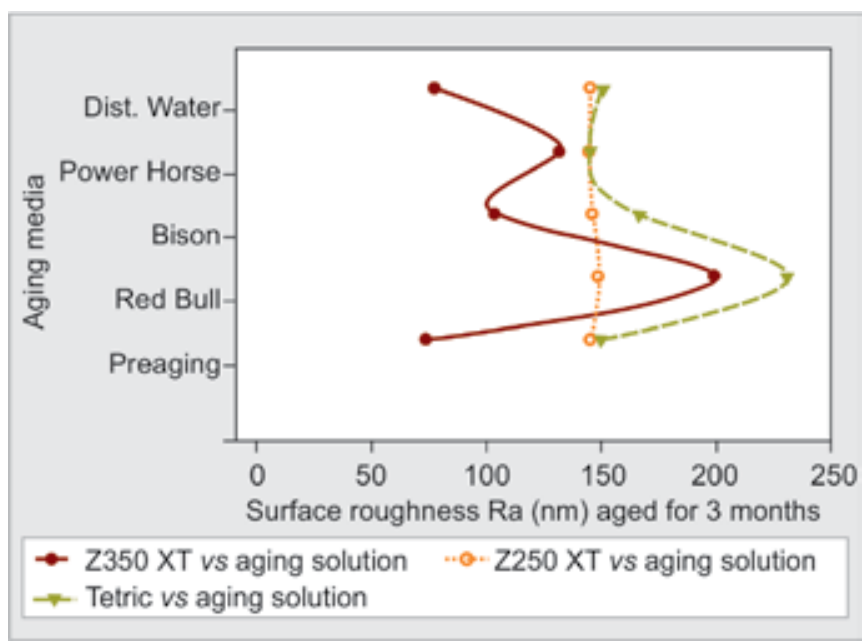

Fig. 2: Surface roughness $\mathrm{Ra}(\mathrm{nm})$ parameter pre- and postaged for 3 months with energy drinks for nanofilled composite resin

total surface roughness difference $\Delta \mathrm{Ra}$ and $\mathrm{Ra}$ after aging with time, however, this was clinically acceptable $(\operatorname{Ra}<0.5 \mu \mathrm{m})$ after 6 months of aging.

The surface roughness $\mathrm{Ra}$ of the three composite specimen materials preaging with the initial measurement, are also different from those postaging with the final measurement $\mathrm{Ra}$, which reflects surface degradation of the materials (Figs 1 to 3).

\section{DISCUSSION}

The improvement of the nanofilled composite resin materials and the demand for esthetic restorations, the composite resin has become the material of choice for anterior teeth restorations. ${ }^{7,12}$ But, despite the improvement of its physical and chemical properties, surface roughness is still a limitation on the longevity of the restoration. ${ }^{7}$

An increase in the demand from patients for improved esthetics has resulted in the development of restorative materials with excellent esthetic properties and their widespread use in dental practice. However, a major disadvantage of resin composites is their tendency to the surface degradation duo to increase surface roughness, which may be a major factor in the replacement of restorations. ${ }^{13}$ Therefore; restorative materials should match well the initial surface properties and preserve the esthetic semblance over time in the restored tooth. ${ }^{14}$

The resin materials are susceptible to surface roughness degradation after immersion in solutions such as sports drinks, cola, acidic juices, coffee and other beverages. However, when referring to the association immersion in solutions, few studies have been carried out to demonstrate the changes in physical properties of composites. ${ }^{7}$ Previous studies have been shown that fillers tend to fall out from resin materials, ${ }^{5}$ and the matrix component decomposes when exposed to low $\mathrm{pH}$ environments. ${ }^{5,15}$ 
This surface degradation phenomenon (fallout of fillers and decomposition of the matrix components) of resin cements. ${ }^{16}$ Many energy and soft drinks are acidic and the $\mathrm{pH}$ is 3.0 or lower. This means that drinking acidic drinks over a long period and with continuous sipping can erode the tooth enamel and the resin material as well.

In the present study, the Surface roughness profile of the three nanofilled resin composites, all of which can be used for anterior and posterior applications in dental practices, was assessed under the actions of acidic energy drinks, which are commonly consumed by the general population. The three resin tested composite materials in this study revealed significant surface roughness changes after 6 months of immersion in the three types of solutions.

The surface roughness $(\mathrm{Ra})$ of the Filtec Z250 XT resin composite was the least affected by the immersion in Power Horse solution, whereas the Filtec Z350 XT resin composite was the most prone to surface roughness Ra change with the Red Bull solution. The increase of the surface roughness $\mathrm{Ra}$ of the composite materials were related to the resin filler type, type of resin matrix, and type of acidic energy drinks and staining agent. ${ }^{17}$ Resin composite materials that can absorb water are also capable of absorbing other acidic fluids with pigments, resulting in surface degradation. It is assumed that water acts as a conductor for the acidic penetration into the resin matrix. ${ }^{18,19}$ Although the resin matrix of the composite materials can absorb water from the environment into the bulk of their structure, inorganic glass fillers cannot absorb water into the bulk of the material, but just absorb water on their surface. Excessive water sorption may decrease the life of a resin composite by expanding and plasticizing the resin component, hydrolyzing the silane, and causing microcrack formation. As a result, the microcracks or interfacial gaps at the interface between the filler and matrix allow surface degradation acid and other liquid solution component as pigment penetration and increase surface roughness $(\mathrm{Ra}){ }^{18}$

Owing to the acidity and erosive potential of energy drinks. ${ }^{20}$ The composite specimens were immersed in the energy (sports) drinks for specified periods of 1, 3 and 6 months to evaluate the surface roughness $(\mathrm{Ra})$ change that can be assessed by specific instruments. The surface quality and susceptibility to external degradation of the energy drinks on the surface profile ( $\mathrm{Ra}$ ), in this experiment was investigated. The same procedure and measurements were carried out on each type of material at the three conditions (aging beverages), using the same equipment. The methodology used in the present study was in accordance with previous studies that used with a surface scanning interferometry (Contour GT-K0 BRUKER - USA) and the $\mathrm{Ra}$ surface roughness parameter were selected from many other parameters surface profile coordinate system, which is a recommended method for dental purposes. ${ }^{14} \mathrm{Ra}$ is the arithmetical mean roughness of all the values of the $\mathrm{Ra}$ profile within the measuring length.

Surface roughness profile coordinate system, which is a recommended method for dental purposes. ${ }^{14}$ The Ra surface roughness parameter coordinate system was chosen to evaluate the surface roughness profile variation because it is well suited for the determination of small roughness changes and has advantages such as repeatability, sensitivity, and objectivity. ${ }^{21}$

It is important to note that surface roughness means measure between 0.5 and $10 \mu \mathrm{m}$ (clinically unacceptable) were sufficient for retaining most bacteria, and are thus not protected against removal forces. $^{22}$

The study of $\Delta \mathrm{Ra}$ and $\mathrm{Ra}$ before and after, values (Table 2) showed that all of the tested materials had values of $\Delta \mathrm{Ra}$ and $\mathrm{Ra}$ parameter which is clinically acceptable and this value is measurable with the help of a surface scanning interferometry.

The Ra surface roughness parameter shift and increase in different test energy drinks was highly significantly different in composites immersed with Red Bull ( $\mathrm{p}<0.000)$, Bison $(\mathrm{p}<0.000)$ and Power Horse $(\mathrm{p}<0.000)$.

When the surface roughness change of the tested materials that immersed in Red Bull in a period of 1, 3 and 6 months was the highest values of the surface roughness Ra parameter among all other materials aged with Bison and Power Horse beverages and highly significant $(p<0.000)$ clinically acceptable, where the highest Ra value was Z350 $\mathrm{XT} \Delta \mathrm{Ra} 144 \mathrm{~nm}$, Tetric EvoCeram $\Delta \mathrm{Ra} 127.8 \mathrm{~nm}$ and the lowest was Z250 XT $\Delta$ Ra $10.73 \mathrm{~nm}$.

$\Delta \mathrm{Ra}$ surface roughness in specimens immersed in Bison for the assigned periods were clinically acceptable in the all periods of aging for the all composite materials Z350 XT revealed $\Delta R$ a surface roughness $43.45 \mathrm{~nm}$ (highest value), Tetric EvoCeram $\Delta \mathrm{Ra} 18.5 \mathrm{~nm}$ and Z250 XT $\Delta$ Ra parameter value $3.83 \mathrm{~nm}$ (the lowest value), where all the materials increases in the $\Delta \mathrm{Ra}$ surface roughness and statistically highly significant $(\mathrm{p}<0.000)$ as tabulated in Table 2 and graphically in Figures 1 to 3 .

The total surface roughness $(\mathrm{Ra})$ difference $\Delta \mathrm{Ra}$ in specimens of all tested materials immersed in Power Horse beverages was also increasing by time and statistically highly significant $(\mathrm{p}<0.000)$. The Ra surface roughness was clinically acceptable in the all periods of aging where $\Delta \mathrm{Ra}$ for the Z350 XT $66.4 \mathrm{~nm}$ was the highest and the lowest was for Z250 XT $\Delta \mathrm{Ra}-3.77 \mathrm{~nm}$, where the acidic effect of this type of energy drinks after the period of 6 months of aging was the least effect among other types as in Table 2 and Figures 1 to 3.

In contrast, the matrix resin of Z250 XT (nanohybrid filler) was hardly exposed between small and large fillers. This suggested that a relatively high filler loading increased 
the stability of the resin composite surface. Z250 XT contains a unique combination of different size and type of nanofiller particles and cluster fillers. The higherfiller content rate was achieved with these types of particles that were mixed. Therefore, Z250 XT of higherfiller contents seemed to have a higher resistance to acidic solutions. This served to explain why these nanohybrid composites showed least surface degradation. ${ }^{5}$

The present study is in agreement with previous findings. ${ }^{5,8,23}$ Our study demonstrated that the tested solutions' induced varying degrees of surface degradation in the resin composites tested after periods of 1,3 and 6 months.

The results of this study as well as of others support the reliability of measurement of surface roughness parameter technology using the surface scanning interferometry system.

\section{CONCLUSION}

With the limitations of this in vitro study, it can be concluded that all energy drink solutions used in this study affected the surface roughness degradation of tested resin composites materials with the increasing of aging time. The effect of energy drinks solutions on the surface roughness parameter of resin composites depends on type of solution and its acidity contents.

\section{REFERENCES}

1. Rajkumar K, Kumar S, Mahalaxmi S, Ragavi P, Mageshwaran TA. Colour stability of resin composites after emersing in coffee of different temperature - an in vitro study. SRM University Journal of Dental Sciences 2011; 2:91-95.

2. Ertan ERTA, et al. Colour stability of resin composites after immersion in different drinks. Dental Materials Journal 2006; 25:371-376.

3. Berger SB, Palialol ARM, Cavalli V, Giannini M. Surface roughness and staining susceptibility of composit resins after finishing and polishing. J Esthet Restor Dent 2011;23:34-45.

4. Higgins JP, Tuttle TD, Higgins CL. Energy beverages: content and safety. Mayo Clin Proc 2010;85(11):1033-1041.

5. Han L, Okamoto A, Fukushima M, Okiji T. Evaluation of flowable resin composite surfaces eroded by acidic and alcoholic drinks. DMJ 2008;27(3):455-465.

6. Tahmassebi JF, Duggal MS, Malik-Kotru G, Curzon MEJ. Soft drinks and dental health: a review of the current literature. J Dent 2006;34:2-11.

7. Lepri CP, Palma-Dibb RG. Surface roughness and color changes of a composite: Influence of beverages and brushing. Dental Material Journal 2012;31(4):689-696.

8. Cochrane NJ, Yuan Y, Walker GD, Shen P, Chang CH, Reynolds C, Renolds EC. Erosive potential of sports beverages. Australian Dental Journal 2012;57:1-6.
9. Ergucu Z, Turkun IS, Aladag A. Color stability of nanocomposites polished with one step system. Operative Dentistry 2008;33: 413-420.

10. Liberman R, Combe EC, Piddock Y, Powson C, Watts DC. Development and assessment of an objective method of color change measurement for acrylic denture base resins. J Oral Rehabil 1995;22(6):445-449.

11. Satou N, Khan AM, Matsumae I, Satou J, Shintani H. In vitro color change of composit based resins. Dent Mater 1989;5: 384-387.

12. Hickle R, Heidemann D, Staehle HJ, Minning P, Wilson NH. Direct composite restorations: extended use in anterior and posterior situations. Clin Oral Investig 2004;8:43-44.

13. Al-Negrish AR. Composite resin restorations: a cross-sectional survey of placement and replacement in Jordan. International Dental Journal 2002;52:461-468.

14. Nasim I, Neelkantan P, Sujeer R, Subbarao CV. Color stability of microfilled, microhybrid and nanocomposite resins - an in vitro study. Journal of Dentistry 2010;38:e137-142.

15. Kitchens M, Owens BM. Effect of carbonated beverages, coffee, sports and high energy drinks and bottled water on the in vitro erosion characteristics of dental enamel. J Clin Pediatr Dent 2007;31:153-159.

16. Neamat AB, Han L, Okamoto A, Iwaku M. Changes in the mechanical properties and surface texture of compomer immersed in various media. J Prosth Dent 2000;84:444-452.

17. Erdemir U, Yildiz E, Eren MM. Effect of sports drinks on color stability of nanofilled and micro-hybrid composites after long term immersion. Journal of Dentistry 2012;40(2):e55-e63.

18. Bagheri R, Burrow MF, Tyas M. Influence of food simulating solutions and surface finish on susceptibility to staining of aesthetic restorative materials. Journal of Dentistry 2005;33: 389-398.

19. Schulze KA, Marshall SJ, Gansky SA, Marshall GW. Color stability and hardness in dental composites after accelerated aging. Dental Materials 2003;19:612-619.

20. Coombes JS. Sports drinks and dental erosion. American Journal of Dentistry 2005;18:101-104.

21. Brook AH, Smith RN, Lath DJ. The clinical measurement of tooth color and stain. International Dental Journal 2007;57: 324-330.

22. Rahal JS, Mesquita MF, Henriques GEP, Nobilo MAA. Surface roughness of acrylic resins submitted to mechanical and chemical polishing. J Oral Rehabilitation 2004;31:1075-1079.

23. Poggio C, Dagna A, Chiesa M, Colombo M, Scribante A. Surface roughness of flowable resin composite eroded by acidic and alcoholic drink. J Conserv Dent 2012;15(2):137-140.

\section{ABOUT THE AUTHOR}

\section{Khalid H Al-Samadani}

Assistant Professor and Consultant, Department of Restorative Dental Science, College of Dentistry, Taibah University, Al Madinah Al Munawwarah 43353, Kingdom of Saudi Arabia, e-mail: kalsamadani@ yahoo.com 